\title{
MR imaging of perianal fistulas in Crohn's disease: sensitivity and specificity of STIR sequences
}

\author{
Giuseppe Lo $\operatorname{Re}^{1} \cdot$ Chiara Tudisca $^{1} \cdot$ Federica Vernuccio $^{1} \cdot$ Dario Picone $^{1} \cdot$ \\ Maria Cappello $^{2}$ - Agnello Francesco ${ }^{1}$ Massimo Galia ${ }^{1}$ Maria Cristina Galfano ${ }^{1}$. \\ Ennio Biscaldi ${ }^{3} \cdot$ Sergio Salerno $^{1} \cdot$ Antonio Pinto $^{4} \cdot$ Massimo Midiri $^{1}$ \\ Roberto Lagalla ${ }^{1}$
}

Received: 8 July 2015 / Accepted: 3 November 2015

(C) Italian Society of Medical Radiology 2015

\begin{abstract}
Introduction Although some studies proved the role of STIR sequences in the evaluation of perianal fistulas in Crohn's Disease (CD), contrast medium is still injected in many institutions since there is not a validated reference MR protocol. Our purpose was to evaluate the role of the STIR sequence in the detection and characterization of perianal fistulae comparing it to the post-contrast $\mathrm{T} 1$ sequence and correlating it with rectal examination under anesthesia. Materials and methods We retrospectively reviewed all clinical records of $31 \mathrm{CD}$ patients, suspected of having perianal fistulas, who had been submitted to an MR study before and after contrast medium injection and surgical exploration under anesthesia within the same month. Perianal fistulas were classified according to the Parks' criteria. Finally, comparison between STIR and post-contrast T1-weighted fat saturated sequences was done.

Results 29 fistulas were detected in 25 patients who underwent an MR study. There was no significant difference between MR imaging and exploration under anesthesia. For the detection of perianal fistulas of any type, there was a perfect statistical agreement between
\end{abstract}

Federica Vernuccio

federicavernuccio@gmail.com

1 Radiology Section, DIBIMED. University of Palermo, Via del Vespro 129, 90127 Palermo, Italy

2 Gastroenterology and Hepatology Section, Di.Bi. Mis., University of Palermo, Piazza Delle Cliniche 2, 90127 Palermo, Italy

3 Department of Radiology, Galliera Hospital, Via Mura delle Capuccine 14, 16128 Genoa, Italy

4 Department of Radiology, Cardarelli Hospital, Naples, Italy gadolinium-enhanced and STIR sequences (kappa value $=1$ ).

Conclusion STIR sequences represent a valid alternative to the T1-weighted sequences acquired after the injection of contrast medium, allowing the identification of the primary fistula, any secondary ramification, and complications of the disease.

Keywords Inflammatory bowel disease $\cdot$ Crohn's Disease $\cdot$ Magnetic resonance imaging $\cdot$ STIR

\section{Introduction}

Perianal fistulas are one of the most unfortunate complications of Crohn's Disease (CD) leading to anal pain, discharge and incontinence, and impairing patients' social life. The lifetime risk for developing a fistula in $\mathrm{CD}$ patients is approximately $25-50 \%[1,2]$, while recto-vaginal fistulas accounted for $9 \%$ [3]. The development of perianal fistulas can result in considerable morbidity and inaccurate diagnosis before treatment may lead to irreversible functional consequences.

Assessment of perianal CD is still challenging. The surgical examination under anesthesia (EUA) has been considered the gold standard for assessing fistulas for years and its tested accuracy is up to $90 \%[4,5]$. Fistulography [6] and pelvic computed tomography (CT) [7, 8] are unlikely to be sufficiently accurate.

Pelvic magnetic resonance imaging (MRI) is a highly accurate non-invasive modality for the diagnosis and classification of perianal fistulas [9].

According to the recent European Crohn's and Colitis Organization (ECCO) guidelines [10], MRI surpassed (EUA) in terms of accuracy and is recommended during 
the initial diagnosis of perianal CD unless there is a need for immediate drainage of sepsis. MRI proved to be accurate in the achievement of an optimal definition of the pelvic anatomy and in the detection and classification of superficial and deep fistulas and their relation to surrounding structures [11-13]. Most MRI protocols routinely use intravenous gadolinium contrast-enhanced images for the evaluation of perianal $\mathrm{CD}$, and more recently subtraction techniques have been used as well as dynamic imaging [7, 14].

Since 1998, STIR (Short-Tau Inversion Recovery) sequences, which do not require contrast medium injection, represent a proved useful tool in the classification of fistulain-ano and in the assessment of the anatomy of perianal fistulas. However, contrast medium is still used to assess perianal CD in different radiological centers as demonstrated by many studies [15-17]. Recent guidelines reported the usefulness of gadolinium-enhanced T1-weighted sequence for the differentiation between fluid/pus and granulation tissue [18].

However, the use of contrast medium involves higher costs and possible adverse drug reactions [19, 20]; moreover, it is contraindicated in some patients.

This study was designed to determine the accuracy of MRI using STIR and post-contrast T1-weighted FSE in assessing suspected $\mathrm{CD}$ perianal fistulas relating the findings of these sequences to rectal EUA.

\section{Materials and methods}

\section{Study population}

We retrospectively reviewed all clinical records of patients with a definite diagnosis of CD followed up in our IBD clinic (which is a tertiary referral center for gastroenterological disease), from January 2010 to December 2013.

Patients were included in the study if they had a definite diagnosis of small bowel CD based on clinical, endoscopic and histological findings, had been submitted to pelvic $M R I$ for the evaluation of perianal $C D$ and studied with surgical EUA within 1 month since MRI study. No patients had undergone a previous surgical procedure for their perianal $\mathrm{CD}$.

Exclusion criteria were age below 18 years, pregnancy, contraindications to pelvic MRI (as implanted metal devices or documented allergic reaction to contrast medium), and renal insufficiency.

Of the 137 patients who performed pelvic MRI to evaluate perianal CD from January 2010 to December 2013, just 31 ( 16 males, mean age 37.2 years, range $20-58$ years) had undergone surgical EUA within the same month and were enrolled in the study.
The study was a retrospective, double blind comparison of the usefulness of STIR and gadolinium-enhanced MRI sequences in the detection of fistulas using rectal EUA as the gold standard. One experienced colorectal surgeon using rectal EUA as the gold standard. Only those patients who had undergone surgical EUA and pelvic STIR and gadolinium-enhanced MRI within the same month were considered to ensure no changes in fistula anatomy between the assessments of the different diagnostic modalities. A fistula anatomy recording form, indicating the number and location of fistulas, abscesses and other findings, was completed by the radiologist and the surgeon. The interpretations of MRI studies were compared to the definitive surgical findings.

As standard protocol of our institution, all patients were asked to sign an informed consent to use their personal data and every patient also had to read an informative report about the tutelage of the personal data. Statistical analyses were done after removing personal data according to the law.

The present project is a retrospective study and so it is not subject to the directive 2001/20/CE of the European Parliament regulating the application of a good clinical practice during clinical experimentation (Art. 1 comma 1; Art 2/c) and so it was not necessary to require an Ethics Committee ruling.

\section{MRI technique}

All the MRI was performed with an 1.5 Tesla superconducting magnet (GE Medical Systems, Milwaukee, WI, USA) coupled with an external eight-channel pelvic phased-array coil. The patients entered the magnet headfirst, lying supine. The pre-contrastographic sequences were obtained in the three orthogonal imaging planes (axial, coronal, and sagittal) using a fast-spin echo (FSE) pulse sequence. T2-weighted fast relaxation (FR) FSE images (repetition time [TR] $7140 \mathrm{~ms}$, echo time [TE] $127.4 \mathrm{~ms}$-duration $3.51 \mathrm{~min}$ ) were acquired, without fat saturation (FAT-SAT) pulses, in the axial plane. These were used to define the sphincter anatomy and the fistula location. We also acquired sagittal T2-weighted FR FSE FAT-SAT imaging (TR $6440 \mathrm{~ms}$, TE $127.4 \mathrm{~ms}$-duration $2.44 \mathrm{~min}$ ). Both sequences were used with a standardized field of view (FOV) of $24 \times 24 \mathrm{~cm}$ and a matrix of $416 \times 224$. T1-weighted FSE images were acquired in the axial (TR $460 \mathrm{~ms}$, TE $10.7 \mathrm{~ms}$, matrix $416 \times 224$-duration $4.25 \mathrm{~min}$ ) and sagittal (TR $600 \mathrm{~ms}$, TE $11.9 \mathrm{~ms}$, matrix $416 \times 192$-duration $4.4 \mathrm{~min})$ planes. STIR sequences were also obtained in the axial, coronal, and sagittal planes (TR $4920 \mathrm{~ms}$, TE $53.5 \mathrm{~ms}$, inversion time [TI] $130 \mathrm{~ms}-$ duration 8.42\2.38\5.30 min respectively). 
Moreover, we obtained post-contrastographic T1-weighted FSE sagittal, axial and coronal images, with and without fat suppression (TR $600 \mathrm{~ms}$, TE $11.8 \mathrm{~ms}$ ), after injection of $0.1 \mathrm{mmol} / \mathrm{kg}$ body weight of gadobutrol (Gadovist, Bayer, Leverkusen, Germany).

\section{Image analysis}

The images were independently interpreted by two experienced radiologists in pelvic MRI. Both radiologists were blinded to the results of all other imaging studies. The site of abnormality was classified radially around a clock face, with the patient in the lithotomy position. Fistulas were classified according to the Parks' criteria [16] as follows: superficial (a), inter- (b), trans- (c), supra- (d) or extra- (e) sphincteric. Patients were also classified as having simple fistulas or complex perianal disease. A simple fistula was defined as a superficial, inter-sphincteric, or low transsphincteric fistula that has only one opening and is not associated with an abscess and/or is not connected to an adjacent structure such as the vagina or bladder. In contrast, a complex fistula was defined as the higher one (i.e., high trans-sphincteric, extra-sphincteric or supra-sphincteric), has multiple openings, horseshoeing, is associated with a perianal abscess, and/or connects to an adjacent structure such as vagina [17]. The location of the enteric communication (i.e., the internal opening) was recorded with respect to a clock face and its level was recorded as either anal or rectal. A horseshoe extension was defined as any extension from the primary track that appeared to extend to both sides of the internal opening; its presence and location (inter-sphincteric, infralevator, supralevator) were recorded. Moreover, the presence and anatomic location of any abscesses were recorded as follows: superficial, intersphincteric or supralevator.

\section{Surgical EUA}

One experienced colorectal surgeon evaluated the patients and performed the EUA. Previous studies established that an EUA alone is imperfect [4, 18, 19]. The surgeon was blind to MRI examinations when he did his assessment. Then, MRI results were given to the surgeon at the end of the examination, after his assessment (while the patient was still under anesthesia) to maximize his chance of detecting fistula tracks and extensions. Setons (i.e., surgical threads used to drain regions of infection) were placed if appropriate.

\section{Statistical analysis}

The primary endpoint for the study was evaluating MRI findings. The primary analyses were aimed at testing the diagnostic performance of each modality, evaluating the correct classification of fistulas according to the EUA. To explore univariate associations in the distribution of categorical data, the Chi squared test or Fisher's exact test was used, as appropriate. A $P$ value of less than 0.05 was considered statistically significant. For each type of fistula, the number of true-positive, true-negative, false-positive and false-negative diagnoses for both imaging techniques and EUA was also assessed, so that sensitivity (95\% confidence interval, $\mathrm{CI}$ ) could be determined. In addition, fistula classification using STIR and gadolinium-enhanced sequences was compared. The agreement was assessed using the kappa statistic test with $95 \%$ CI (a chance adjusted measure of agreement, $1.0=$ perfect agreement). Analysis was carried out using the software Statistical Analysis System (SAS Institute, Inc., Cary, NC, USA).

\section{Results}

Considering the 31 patients enrolled in the study, fistulas were detected in 25 patients who underwent STIR and gadolinium-enhanced MRI examinations (Table 1).

Eight patients had simple fistulas and 17 had complex perianal disease. According to the Parks' classification, 2 fistulas were superficial, 8 inter-sphincteric, 18 transsphincteric and 1 was extra-sphincteric. Furthermore, in seven cases we found a horseshoe fistula and in six an internal opening. In six cases, MRI did not show any lesion. Eight patients also had a total of nine abscesses (five superficial, four supralevator) and all were correctly identified by both STIR and gadolinium-enhanced MRI compared to EUA (Table 1; Fig. 1). The number of fistula tracks detected according to the different diagnostic modality is shown in Table 2.

False-positive diagnoses were made with MRI in two patients $(6.4 \%)$ who presented with posterior perianal pain. These patients were diagnosed with a type B fistula based on imaging, but surgical EUA did not reveal any fistula. These patients had a previous fistula that had healed 1 year before, leaving a fibrotic track. False-negative diagnose occurred with MRI in one patient who was diagnosed with having no fistula at imaging, but surgical EUA revealed a type A fistula. MRI correctly found out the main track type and the presence of all complex fistulas, including the internal opening and extensions. Table 3 shows the sensitivity of STIR, gadolinium-enhanced MRI and surgical EUA in detecting fistulas and abscesses.

Imaging was less sensitive than EUA only for the detection of superficial fistulas. There was no significant difference between MRI and EUA in the recognition of complex fistulas, internal opening and extension. For the detection of perianal fistulas of any type, there was a perfect 
Table 1 Diagnostic yield of magnetic resonance imaging techniques

\begin{tabular}{|c|c|c|c|c|}
\hline & \multicolumn{2}{|l|}{ STIR } & \multicolumn{2}{|c|}{ Gadolinium-enhanced MRI } \\
\hline & Number of patients & Number of lesions & Number of patients & Number of lesions \\
\hline \multicolumn{5}{|l|}{ Fistula type* } \\
\hline Superficial & 2 & 2 & 2 & 2 \\
\hline Inter-sphincteric & 6 & 8 & 6 & 8 \\
\hline Trans-sphincteric & 16 & 18 & 16 & 18 \\
\hline Supra-sphincteric & - & - & - & - \\
\hline Extra-sphincteric & 1 & 1 & 1 & 1 \\
\hline Total $^{\mathrm{a}}$ & 25 & 29 & 25 & 29 \\
\hline Horseshoes & 7 & 7 & 7 & 7 \\
\hline Internal openings & 6 & 6 & 6 & 6 \\
\hline Abscesses & 9 & 9 & 9 & 9 \\
\hline Superficial & 4 & 5 & 4 & 5 \\
\hline Supralevator & 4 & 4 & 4 & 4 \\
\hline Total $^{\mathrm{b}}$ & 8 & 9 & 8 & 9 \\
\hline
\end{tabular}

STIR Short-Tau Inversion Recovery, MRI magnetic resonance imaging

* According to the Parks' classification

a Some patients had more than one fistula and are thus counted more than once

${ }^{\mathrm{b}}$ One patients had more than one abscesses and is thus counted more than once
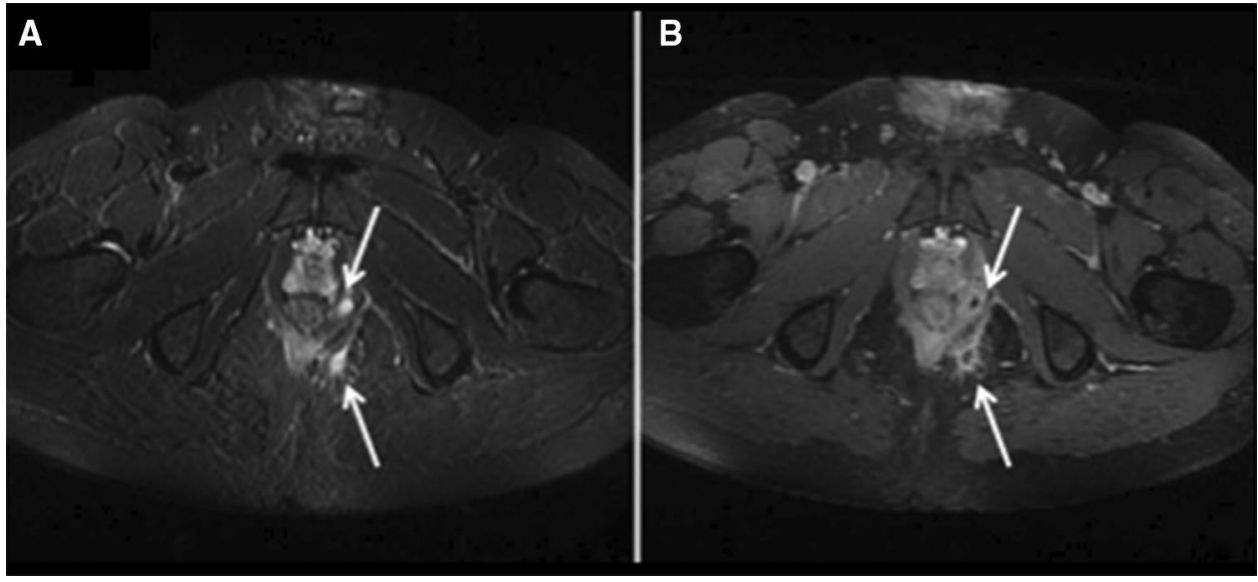

statistical agreement between gadolinium-enhanced and STIR sequences (Figs. 2; 3), with kappa values (95 \% CI) of 1 . There was no statistical difference in detecting and evaluating abscesses between imaging findings and surgery EUA; moreover, all abscess was easily detected both using STIR and post-contrastographic T1-weighted sequences. The agreement between the MRI techniques and EUA showed a kappa value $(95 \% \mathrm{CI})$ of $0.870(0.735-1.0)$.

\section{Discussion}

Our study outlines the usefulness of MRI in recognizing the extension of the primary anal fistulous tracts in CD patients through STIR post-contrast sequences, proving a comparable sensibility in their diagnosis.
The perianal involvement in patients with $\mathrm{CD}$ is quite frequent. Anorectal complications develop in $36 \%$ of all patients with $\mathrm{CD}$, particularly in the $25 \%$ with only small bowel involvement, in $67 \%$ of patients with colonic CD and in almost all patients with rectal involvement [9]. In 36-81\% of cases, the development of anorectal disease may precede or occur simultaneously with ileo-cecal manifestation and it can be rarely identified as the only clinical manifestation of the disease [7].

The presence of perianal disease, especially at the time of diagnosis, is a negative prognostic factor with greater risk of progression to surgery [14]. Several imaging modalities, as the Endoanal Ultrasound, fistulography, CT and MRI, allow the visualization of perianal fistulas [6-8, 21-29]. Among these, endoanal ultrasound provides a good 
Table 2 Univariate analysis of surgical examination and magnetic resonance imaging findings

$\begin{array}{ll}\text { Correctly classified condition } & k \text { test } \\ \text { STIR/gadolinium-enhanced EUA } & \\ \text { MRI } & \end{array}$

Fistula type $(n=30)$

\begin{tabular}{llll} 
Superficial & 2 & 3 & 1 \\
Inter-sphincteric & 8 & 6 & 0.7 \\
Trans-sphincteric & 18 & 18 & 0.7 \\
Supra-sphincteric & - & - & - \\
Extra-sphincteric & 1 & 1 & 0.4 \\
Horseshoes $(n=7)$ & 7 & 7 & 0.7 \\
Internal openings $(n=6)$ & 6 & 6 & 0.7 \\
Abscesses $(n=9)$ & 9 & 9 & 0.8 \\
\hline
\end{tabular}

Data are given as number of lesions

STIR Short-Tau Inversion Recovery, MRI magnetic resonance imaging, EUA exploration under anesthesia

Table 3 Sensitivity of magnetic resonance imaging with regard to the fistula type

Sensitivity $(95 \% \mathrm{CI})$

\begin{tabular}{lc}
\hline Fistula type & \\
Superficial & $66.6 \%(0.125-0.982)$ \\
Inter-sphincteric & $100 \%(0.516-1)$ \\
Trans-sphincteric & $100 \%(0.781-1)$ \\
Extra-sphincteric & $100 \%(0.054-1)$ \\
Horseshoes & $100 \%(0.560-1)$ \\
Internal openings & $100 \%(0.516-1)$ \\
\hline
\end{tabular}

MRI magnetic resonance imaging, EUA exploration under anesthesia, STIR Short-Tau Inversion Recovery

visualization of the intersphincteric plane and the perianal and perirectal soft tissue and is used in the assessment of patients with suspected perianal sepsis, but cannot distinguish areas of fibrosis from the active areas of inflammation and is inadequate for evaluation of ischioanal fossa and supraelevator region $[21,24]$. The surgical physical examination has been considered the "gold standard" (sensitivity $99.8 \%$ ) for the identification of perianal fistulas in CD for years [7]. Actually, MRI has an increasingly important role in the evaluation of disease activity in CD and of complicated CD and is actually considered the gold standard and recommended during the initial diagnosis of perianal $\mathrm{CD}$ according to the ECCO guidelines; moreover, compared to endoanal ultrasound it is comparable for the evaluation of perianal fistulas and proved to be superior for discriminating between simple and complex disease compared to clinical assessment and anal endosonography [10, 30-35].
MRI allows an accurate analysis of the fistulas with a correct view of their course, their full extension, and especially the relationship between them, the anal sphincter complex and the surrounding pelvic structures. In particular, the MRI on the axial and coronal planes allows the identification of the connection between the fistula and the sphincter apparatus, the levator ani muscle and the ischiorectal fossa; images on the sagittal plane are useful in the evaluation of rectovesical and rectovaginal fistulas, and in the evaluation of the presacral disease $[11,13]$.

According to the literature, in our MRI examinations we used an eight-channel surface [36-38].

The most used sequence in different studies is the T2-weighted FSE, but in others studies T1-weighted FSE sequences before and after intravenous administration of contrast medium are used [15-18, 36, 39, 40].

The techniques with suppression of the fat signal are applied to both T1-weighted and T2-weighted sequences [41].

In a recent study, Singh et al. [16] stated that the contrast study can be omitted, particularly while evaluating primary/previously unoperated perianal fistulae because the results of imaging findings on T2-weighted and postcontrast $\mathrm{T} 1$-weighted fat saturated sequences were statistically similar. In our study, we focused on the role of STIR sequences in patients with $\mathrm{CD}$ in the diagnosis of perianal fistulas and in the evaluation of the extension of the inflammatory process in the perianal and perirectal areas, by comparing STIR and T1-images.

STIR sequences are usually used in the study of anatomical regions where there is a substantial fat content and are an important application of the inversion recovery sequences [42].

The visualization of the fistulas is related to the degree of inflammation and consequently to their content. The signal suppression of adipose tissue obtained thanks to STIR sequences provides a good contrast between fistula and adjacent tissues and makes easier its identification [42-44]. In the active phases of the disease, the fistula shows both walls containing granulation tissue and fluid or mucin in its lumen; furthermore, it presents itself as a stria or as a band of tissue with high signal intensity, well contrasted compared to muscles and surrounding fat [45]. On T1-weighted images, acquired with fat suppression and after the use of paramagnetic contrast agents (gadolinium and its chelates), the walls of fistulas-containing granulation tissue-as well as the areas of active inflammation, show intense postcontrastographic enhancement [21, 38, 45].

The fistulas in the active phase are often surrounded by hypointense fibrous walls, which can be relatively thin, particularly in patients with recurrent disease, or with a recent surgical operation; however, in case of edema, the walls 
Fig. 2 Axial STIR (a) and T1-weighted post-contrast (b) images show that a fistula (arrow) originates from the left postero-lateral wall of the anal canal (type $\mathrm{C}$ according to Parks classification). The same sequences acquired on the coronal plane (c and d) allow a better assessment of the fistula (arrowhead) and its relationship with the ischio-rectal fossa
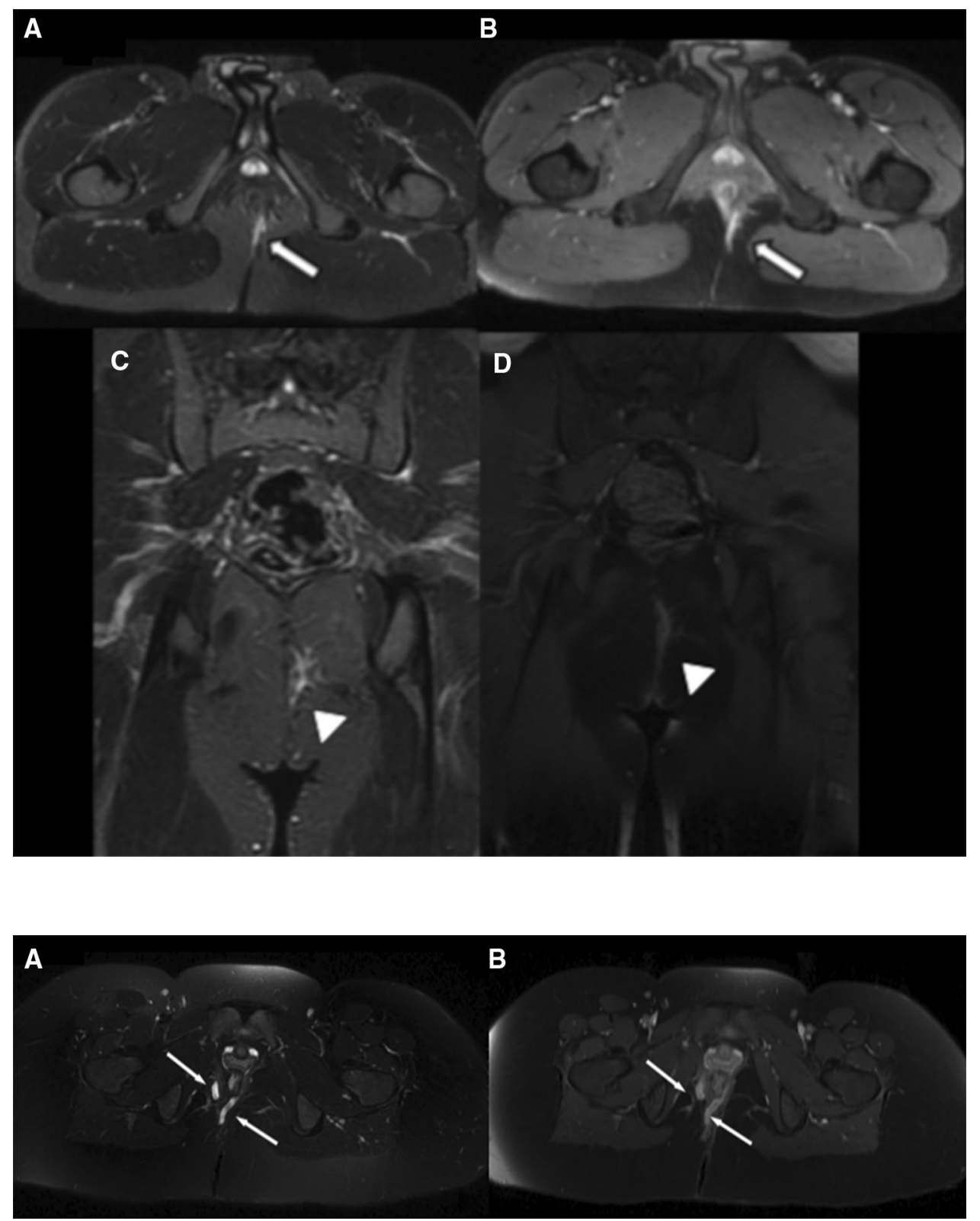

Fig. 3 Axial STIR images (a) and post-contrast $\mathrm{T} 1$-weighted (b) images show the presence of two transphincteric fistulas (type C of Park's) with contiguous little inflammatory component (arrows). There is a perfect agreement between the findings on the STIR and post-contrast T1-weighted images may appear hyperintense and this hyperintensity can spread into the adjacent parenchyma.

The external anal sphincter is moderately hypointense and its wall contrasts with the fat of the ischio-anal fossa, both in STIR and FAT-SAT sequences. It is, therefore, relatively easy to distinguish the presence of a fistula in it. However, misdiagnosis due to false-positive reports can occur in patients recently submitted to surgery because of the high inhomogeneity of the perianal tissue (Fig. 4).

The main advantage of the MRI, as underlined by our results, lies in how it can easily recognize the extension of the primary fistulous tract, which is hyperintense both on T2-weighted and STIR sequences, and shows contrast enhancement. It is also important to recognize the relationships with the levator ani muscle and the extensions above it.

In our study, the comparison of imaging findings between the two different types of sequences did not show any significant difference. The findings reported with STIR technique are similar to those disclosed using post-contrastographic T1-weighted images.

Indeed, the number of the fistulas and their densitometric and morphological characteristics identified in the 31 patients enrolled in our study using STIR sequences were confirmed by T1-weighted images acquired after administration of contrast medium.

We also compared MRI and surgical visit findings, showing a sensitivity of the method of $92 \%$. 


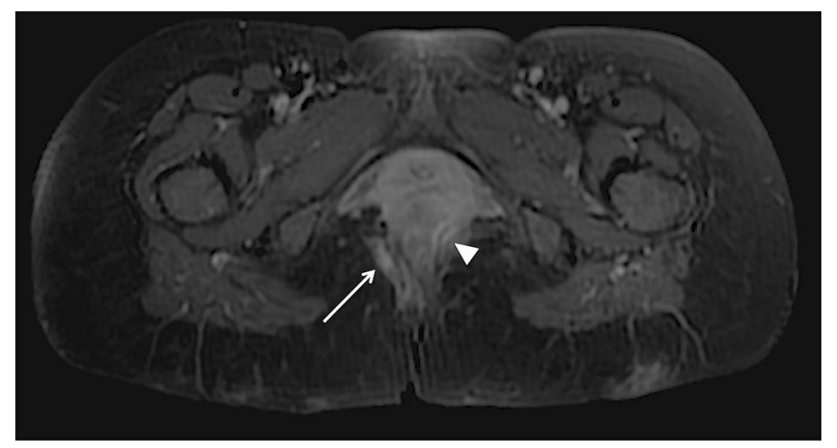

Fig. 4 Axial STIR image (a) and post-contrast T1-weighted (b) images show the presence of active transphincteric fistulas (type $\mathrm{C}$ of Park's) in the posterior right side of the perianal tissue (arrow) that was confirmed at EUA, and another one in the left posterior side that was not confirmed (arrowhead)

Both techniques are effective in the diagnosis of perianal fistula associated with $\mathrm{CD}$, providing information on the course and extension of the fistula and on its relationship with the anal sphincter and on the presence of any abscesses. All this information is necessary for a proper preoperative evaluation of the patients and for the most appropriate surgical planning [46-48].

Recent works emphasize the ability of the STIR sequence of highlighting the presence of pus and granulation tissue without the use of contrast medium, but stress the need to obtain T1-weighted sequence after contrast to get anatomical details required for classification of the fistula [11, 45, 48].

However, other studies demonstrated the possibility of recognizing and classifying the fistula using only the STIR sequences [25, 49], as we also demonstrated.

The limitations of the present study are as follows. First, this study is a retrospective assessment. However, we consecutively enrolled every participant submitted to pelvic MRI to evaluate perianal CD from January 2010 to December 2013, and collected the hospitalization medical records, images and reports as completely as possible. Second, this study was undertaken in a single medical institution and, therefore, the number of patients studied was limited. The limited number of patients is not adequate for a reliable statistical analysis and may lead to overestimated values of accuracy. Third, the radiologists evaluated the two types of MRI sequence, and information of the first sequence would influence interpretation of the second. However, radiologists were blinded to the results of EUA and images of the different sequences of the different patients were viewed randomly by the radiologists.

\section{Conclusion}

Our study showed the value of STIR sequences in the identification and classification of perianal fistulas and their complications. The use of these sequences provided results similar to those obtained through T1-weighted images acquired after the administration of gadolinium contrast agents. Therefore, STIR sequences represent a valid alternative to the SE T1-weighted FAT-SAT sequences acquired after the injection of contrast medium, allowing the identification of the primary fistula, of any secondary ramifications, of abscesses, of the involvement of the anal sphincter, the levator ani and of the ischium-anal and ischium-rectal fissures.

The advantage of using the STIR sequences lies in the possibility of obtaining the same diagnostic content of T1-weighted images acquired after infusion of gadolinium, but without the use of the latter, thus avoiding the possible adverse drug reaction and renal function impairment related to its use. Moreover, MRI is a minimally invasive radiation free technique that allows to obtain a lot of diagnostic information with the minimum "discomfort" for the patient, with a very high sensitivity of detection of perianal disease, as we reported in our study.

\section{Compliance with ethical standards}

Conflict of interest Giuseppe Lo Re declares that he has no conflict of interest, Chiara Tudisca declares that she has no conflict of interest, Federica Vernuccio declares that she has no conflict of interest, Dario Picone declares that he has no conflict of interest, Maria Cappello declares that she has no conflict of interest, Agnello Francesco declares that he has no conflict of interest, Massimo Galia declares that he has no conflict of interest, Maria Cristina Galfano declares that she has no conflict of interest, Ennio Biscaldi declares that he has no conflict of interest, Sergio Salerno declares that he has no conflict of interest, Antonio Pinto declares that he has no conflict of interest, Massimo Midiri declares that he has no conflict of interest, Roberto Lagalla declares that he has no conflict of interest.

\section{Funding None.}

Ethical approval The present project is a retrospective observational study. For this type of study, formal consent is not required.

Informed consent All patients enrolled were asked to sign an informed consent in accordance with the dispositions from the Italian law 675/96, and every patient also had to read an informative report about the tutelage of the personal data, according to the same law. All the patients were asked to sign an informed consent to use the images of their radiological exams for research, according to the law.

Ethical standards This article does not contain any studies with human participants or animals performed by any of the authors. This is a retrospective observational study.

\section{References}

1. Chiari H (1878) Uber die analen divertikel der rectumschleimahut und ihre beziehung zu den anal fisteln. Wien Med Press 19:1482-1483

2. Mc Coll I (1967) The comparative anatomy and pathology of anal glands. Arris and Gale lecture delivered at the Royal 
College of Surgeons of England on 25th February 1965. Ann R Coll Surg Engl 40:36-67

3. Parks AG, Gordon PH, Hardcastle JD (1976) A classification of fistula-in-ano. Br J Surg 63:1-12

4. Langman JM, Rowland R (1992) Density of lymphoid follicles in the rectum and at the anorectal junction. J Clin Gastroenterol 14:81-84

5. Buchan R, Grace RH (1973) Anorectal suppuration: the results of treatment and the factors influencing the recurrence rate. Br J Surg 60:537-540

6. Pomerri F, Dodi G, Pintacuda G et al (2010) Anal endosonography and fistulography for fistula-in-ano. Radiol Med 115:771-783

7. Sandborn WJ, Fazio VW, Feagan BG, Hanauer SB, American Gastroenterological Association Clinical PracticeCommittee (2003) AGA technical review on perianal Crohn's disease. Gastroenterology 125:1508-1530

8. Tonolini M, Villa C, Campari A, Ravelli A, Bianco R, Cornalba G (2013) Common and unusual urogenital Crohn's disease complications: spectrum of cross-sectional imaging findings. Abdom Imaging 38:32-41

9. O'Donovan AN, Somers S, Farrow R, Mernagh JR, Sridhar S (1997) MR imaging of anorectal Crohn disease: a pictorial essay. Radiographics 17:101-107

10. Panes J, Bouhnik Y, Reinisch W, Stoker J, Taylor SA, Baumgart DC, Danese S, Halligan S, Marincek B, Matos C, Peyrin-Biroulet L, Rimola J, Rogler G, van Assche G, Ardizzone S, Ba-Ssalamah A, Bali MA, Bellini D, Biancone L, Castiglione F, Ehehalt R, Grassi R, Kucharzik T, Maccioni F, Maconi G, Magro F, Martín-Comín J, Morana G, Pendsé D, Sebastian S, Signore A, Tolan D, Tielbeek JA, Weishaupt D, Wiarda B, Laghi A (2013) Imaging techniques for assessment of inflammatory bowel disease: joint ECCO and ESGAR evidence-based consensus guidelines. J Crohns Colitis 7:556-585

11. Gage KL, Deshmukh S, Macura KJ, Kamel IR, Zaheer A (2013) MRI of perianal fistulas: bridging the radiological-surgical divide. Abdom Imaging 38:1033-1042

12. Llauger J, Palmer J, Pérez C, Monill J, Ribé J, Moreno A (1998) The normal and pathologic ischiorectal fossa at CT and MR imaging. Radiographics 18:61-82

13. Sahni VA, Ahmad R, Burling D (2008) Which method is best for imaging of perianal fistula? Abdom Imaging 33:26-30

14. Ingle SB, Loftus EV Jr (2007) The natural history of perianal Crohn's disease. Dig Liver Dis 39:963-967

15. Baskan O, Koplay M, Sivri M, Erol C (2014) Our experience with MR imaging of perianal fistulas. Pol J Radiol 79:490-497

16. Singh K, Singh N, Thukral C, Singh KP, Bhalla V (2014) Magnetic resonance imaging (MRI) evaluation of perianal fistulae with surgical correlation. J Clin Diagn Res 8:RC01-RC04

17. Garros A, Siproudhis L, Tchoundjeu B, Rohou T, Brochard C, Wallenhorst T, Bretagne JF, Bouguen G (2014) Magnetic resonance imaging and clinical assessments for perianal Crohn's disease: gain and limits. Dig Liver Dis 46:1072-1076

18. Gecse KB, Bemelman W, Kamm MA, Stoker J, Khanna R, Ng SC, Panés J, van Assche G, Liu Z, Hart A, Levesque BG, D’Haens G, World Gastroenterology Organization, International Organisation for Inflammatory Bowel Diseases IOIBD, European Society of Coloproctology and Robarts Clinical Trials, World Gastroenterology Organization International Organisation for Inflammatory Bowel Diseases IOIBD European Society of Coloproctology and Robarts Clinical Trials (2014) A global consensus on the classification, diagnosis and multidisciplinary treatment of perianal fistulising Crohn's disease. Gut 63:1381-1392
19. Li A, Wong CS, Wong MK, Lee CM, Au Yeung MC (2006) Acute adverse reactions to magnetic resonance contrast mediagadolinium chelates. Br J Radiol 79:368-371

20. Fakhran S, Alhilali L, Kale H, Kanal E (2015) Assessment of rates of acute adverse reactions to gadobenate dimeglumine: review of more than 130,000 administrations in 7.5 years. AJR 204:703-706

21. Brillantino A, Iacobellis F, Di Sarno G, D’Aniello F, Izzo D, Paladino F, De Palma M, Castriconi M, Grassi R, Di Martino N, Renzi A (2015) Role of tridimensional endoanal ultrasound (3D-EAUS) in the preoperative assessment of perianal sepsis. Int J Colorectal Dis 30:535-542

22. Reginelli A, Mandato Y, Cavaliere C, Pizza NL, Russo A, Cappabianca S, Brunese L, Rotondo A, Grassi R (2012) Three-dimensional anal endosonography in depicting anal-canal anatomy. Radiol Med 117(5):759-771. doi:10.1007/s11547-011-0768-4 (Epub 2012 Jan 7. PubMed PMID: 22228126)

23. Brunese L, Amitrano M, Gargano V, Vallone G, Grassi R, Rotondo A, Smaltino F (1996) Anal endosonography: the study technic and the correlations between the normal and echographic anatomy. Radiol Med 91(3):253-257 (Italian. PubMed PMID:8628939)

24. Halligan S, Stoker J (2006) Imaging of fistula in ano. Radiology 239:18-33

25. Lo Re G, Cappello M, Tudisca C, Galia M, Randazzo C, Craxì A, Cammà C, Giovagnoni A, Midiri M (2014) CT enterography as a powerful tool for the evaluation of inflammatory activity in Crohn's disease: relationship of CT findings with CDAI and acute-phase reactants. Radiol Med 119:658-666

26. Minordi LM, Scaldaferri F, Larosa L, Marra R, Giordano F, Laterza L, Scoleri I, Poscia A, Gerardi V, Bruno G, Gaetani E, Gasbarrini A, Vecchioli A, Bonomo L (2015) Comparison between clinical and radiological evaluation before and after medical therapy in patients with Crohn's disease: new prospective roles of CT enterography. Radiol Med 120:449-457

27. Siddiqui MR, Ashrafian H, Tozer P, Daulatzai N, Burling D, Hart A, Athanasiou T (2012) Phillips RK A diagnostic accuracy metaanalysis of endoanal ultrasound and MRI for perianal fistula assessment. Dis Colon Rectum 55:576-585

28. Sun MR, Smith MP, Kane RA (2008) Current techniques in imaging of fistula in ano: three-dimensional endoanal ultrasound and magnetic resonance imaging. Semin Ultrasound CT MR 29:454-471

29. Ardizzone S, Maconi G, Cassinotti A, Massari A, Porro GB (2007) Imaging of perianal Crohn's disease. Dig Liver Dis 39:970-978

30. Foti PV, Farina R, Coronella M, Palmucci S, Ognibene N, Milone P, Conti Bellocchi C, Samperi L, Inserra G, Laghi A, Ettorre GC (2015) Crohn's disease of the small bowel: evaluation of ileal inflammation by diffusion-weighted MR imaging and correlation with the Harvey-Bradshaw index. Radiol Med 120:585-594

31. Lo Re G, Galia M, Bartolotta TV, Runza G, Taibbi A, Lagalla R, De Maria M, Midiri M (2007) Forty-slice TCMD enteroclysis: evaluation after oral administration of isotonic solution in Crohn's disease. Radiol med 112:787-797

32. Sahni VA, Ahmad R, Burling D (2008) Which method is best for imaging of perianal fistula? Abdom Imaging 33:26-30

33. Scholefield JH, Berry DP, Armitage NC, Wastie ML (1997) Magnetic resonance imaging in the management of fistula in ano. Int J Colorectal Dis 12(5):276-279

34. West RL, Dwarkasing S, Felt-Bersma RJ, Schouten WR, Hop WC, Hussain SM, Kuipers EJ (2004) Hydrogen peroxideenhanced three-dimensional endoanal ultrasonography and endoanal magnetic resonance imaging in evaluating perianal fistulas: agreement and patient preference. Eur J Gastroenterol Hepatol 16(12):1319-1324 
35. Gustafsson UM, Kahvecioglu B, Aström G, Ahlström H, Graf W (2001) Endoanal ultrasound or magnetic resonance imaging for preoperative assessment of anal fistula: a comparative study. Colorectal Dis 3(3):189-197

36. Stoker J, Rociu E, Zwamborn AW, Schouten WR, Laméris JS (1999) Endoluminal MR imaging of the rectum and anus: technique, applications and pitfalls. Radiographics 19:383-398

37. Maccioni F, Colaiacomo MC, Stasolla A, Manganaro L, Izzo L, Marini M (2002) Value of MRI performed with phased-array coil in the diagnosis and pre-operative classification of perianal and anal fistulas. Radiol Med 104:58-67

38. Hoeffel C, Arrivé L, Mourra N et al (2006) Anatomic and pathologic findings at external phased-array pelvic MR imaging after surgery for anorectal disease. Radiographics 26:1391-1407

39. Maccioni F, Bruni A, Viscido A, Colaiacomo MC, Cocco A, Montesani C, Caprilli R, Marini M (2006) MR imaging in patients with Crohn disease: value of T2- versus T1-weighted gadolinium-enhanced MR sequences with use of an oral superparamagnetic contrast agent. Radiology 238:517-530

40. Maier AG, Funovics MA, Kreuzer SH, Herbst F, Wunderlich M, Teleky BK, Mittlböck M, Schima W, Lechner GL (2001) Evaluation of perianal sepsis: comparison of anal endosonography and magnetic resonance imaging. J Magn Reson Imaging $14: 254-260$

41. Grieser C, Denecke T, Steffen IG, Werner S, Kröncke T, Guckelberger O, Pape UF, Meier J, Thiel R, Kivelitz D, Sturm A, Hamm B, Röttgen R (2012) Magnetic resonance enteroclysis in patients with Crohn's disease: fat saturated T2-weighted sequences for evaluation of inflammatory activity. J Crohns Colitis 6:294-301
42. Halligan S, Healy JC, Bartram CI (1998) Magnetic resonance imaging of fistula-in-ano: STIR or SPIR? Br J Radiol $71: 141-145$

43. Sabir N, Sungurtekin U, Erdem E, Nessar M (2000) Magnetic resonance imaging with rectal Gd-DTPA: new tool for the diagnosis of perianal fistula. Int J Colorectal Dis 15:317-322

44. Barker PG, Lunniss PJ, Armstrong P, Reznek RH, Cottam K, Phillips RK (1994) Magnetic resonance imaging of fistula-inano: technique, interpretation and accuracy. Clin Radiol 49:7-13

45. Al-Khawari HA, Gupta R, Sinan TS, Prakash B, Al-Amer A, Al-Bolushi $S$ (2005) Role of magnetic resonance imaging in the assessment of perianal fistulas. Med Princ Pract 14:46-52

46. Szurowska E, Wypych J, Izycka-Swieszewska E (2007) Perianal fistulas in Crohn's disease: MRI diagnosis and surgical planning: MRI in fistulazing perianal Crohn's disease. Abdom Imaging 32:705-718

47. Lunniss PJ, Armstrong P, Barker PG, Reznek RH, Phillips RK (1992) Magnetic resonance imaging of anal fistulae. Lancet 340:394-396

48. Haggett PJ, Moore NR, Shearman JD, Travis SP, Jewell DP, Mortensen NJ (1995) Pelvic and perineal complications of Crohn's desease: assessment using magnetic resonance imaging. Gut 36:407-410

49. Szyszko TA, Bush J, Gishen P, Sellu D, Desouza NM (2005) Endoanal magnetic resonance imaging of fistula-in-ano: a comparison of STIR with gadolinium-enhanced techniques. Acta Radio 46:3-8 